\title{
INFRARED ABSORPTION OF MULTIPLE QUANTUM WELLS: BOUND TO CONTINUUM TRANSITIONS
}

\author{
R. OszWaldowsKi and W. Jaskólski \\ Institute of Physies, N. Copernicus University \\ Grudziądzka 5, 87-100 Toruń, Poland
}

\begin{abstract}
Optical transitions between bound and continuum states in multiple-heterojunction quantum structures are numerically investigated. In the conduction band energy range the absorption to continuum is studied within one-band effective-mass approximation. By changing the shape of quantum wells, we can tune the energy corresponding to absorption peak. We also show how the absorption is modified in the case of annealed structures. The inter-subband transitions in the energy range of valence band in $\mathrm{Si} / \mathrm{Si}_{1-x} \mathrm{Ge}_{x} / \mathrm{Si}$ quantum well are described with the help of multiband Luttinger-Kohn Hamiltonian. Particular attention is paid to transitions to resonant states.
\end{abstract}

PACS numbers: 73.20.Dx, 78.66.-w, 85.30.Vw

\section{Introduction}

Bound-to-continuum (B-C) intersubband transitions with a large oscillator strength which occur in semiconductor quantum wells (QWs) are taken advantage of in some infrared photodetectors [1]. In contrast to bound-to-bound (B-B) transitions, the $\mathrm{B}-\mathrm{C}$ ones excite carriers to current conducting continuum states and allow us to employ perpendicular transport (carriers moving across the junctions).

In this paper we show that by appropriate tailoring of QW structures one can substantially modify absorption spectrum yielding neaks with controlled positions. The tailoring can be achieved by growing barrier-acting layers on both sides of well acting material. This leads to appearance of resonant states in the continuum energy spectrum. We study also the influence of annealing process on the intersubband absorption spectra in QW. With increased annealing intensity the wells become both wider and shallower. The interplay of these two trends causes substantial modifications of $B-C$ spectra and binding of new states. We show that in the case of $p$-type $\mathrm{QW}$ resonant states may appear without particular tailoring of the structure. Resonant spectra indicate that it is possible to design selective infrared detectors based on B-C transitions. 


\section{Numerical method}

The bound and continuum states of structures considered in this paper are found variationally using one band effective mass Hamiltonian for electrons and multiband (Luttinger-Kohn) Hamiltonian for holes. The method of calculation of energy levels in QWs we use is an extension of a large box approach presented in Ref. [2]. However, since we calculate B-C transitions we treat the box boundaries as the limits of our quantum system. Thus the continuum becomes discrete and it seems natural to use the standard energy density of states $\rho(E) \approx \sqrt{m^{*} / E}$ to obtain intersubband absorption spectrum. However, employing the above formula for conduction band QWs with additional barriers or simple valence band QWs might lead to inaccuracies caused, for example, by the existence of resonant states in the continuum. Instead, we sum up squared optical transition matrix elements (divided by the photon frequency $\omega$ ) over small intervals of energy. For each such interval the integration of oscillator strengths, weighted by the energy density, and the simple summation are equivalent if performed for the same quantum system size.

\section{Conduction band transitions}

In this section we study the dependence of the $\mathrm{B}-\mathrm{C}$ absorption spectrum profile on the form of $\mathrm{Al}_{x} \mathrm{Ga}_{1-x}$ As QWs. We do this for the case in which both bound and free (scattering) states are derived from the CB.

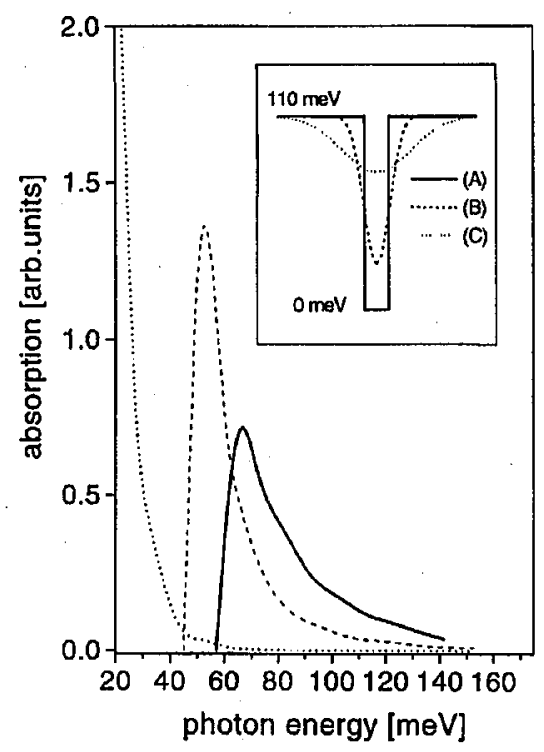

Fig. 1. Bound-to-continuum absorption spectra of three quantum wells: (A) $\mathrm{Al}_{0.15} \mathrm{Ga}_{0.85}$ As QW $6 \mathrm{~nm}$ width (solid line); $(B)$ the same as $(A)$ but after $900^{\circ} \mathrm{C} / 30 \mathrm{~s}$ annealing (dashed line); $(C)$ the same as $(A)$ after $930^{\circ} \mathrm{C} / 60 \mathrm{~s}$ annealing (dotted line). The inset shows conduction band profiles used in the calculation. 
First we study how the process of interdiffusion of heterostructure components, occurring during annealing performed on the sample containing the QW in question, affects the spectrum. Similarly as in Ref. [3] we assume that the position dependence of aluminium molar content $x^{\prime}$ after annealing of a rectangular $\mathrm{Al}_{x} \mathrm{Ga}_{1-x} \mathrm{As}$ quantum well of width $2 L$ is given by a linear combination of error functions, in this case: $x^{\prime}(z)=x\{1-0.5[\operatorname{erf}((L-z) /(2 \sqrt{D t}))$ $+\operatorname{erf}((L+z) /(2 \sqrt{D t}))]\}$, where $D=D_{0} \exp \left(-E_{\mathrm{A}} / k T\right), E_{\mathrm{A}}$ is the activation energy and $t$ is the annealing time. After Ref. [3] we take $D_{0}=427.15 \mathrm{~cm}^{2} / \mathrm{s}$ and $E_{\mathrm{A}}=4.09 \mathrm{eV}$. This value of $E_{\mathrm{A}}$ falls into a wide range of activation energies suggested by other authors $[3,4]$. We use the $4 / 6$ splitting ratio for the VB and the CB which leads to $Q W$ potential profiles shown in the inset of Fig. 1. The first profile $(A)$ corresponds to an as-grown sample with $\mathrm{Al}_{0.15} \mathrm{Ga}_{0.85}$ As quantum well of width $L=6 \mathrm{~nm}$. The second one $(B)$ corresponds to the same $\mathrm{QW}$ annealed at $900^{\circ} \mathrm{C}$ for 30 seconds. The third profile $(C)$ is for $930^{\circ} \mathrm{C} / 60 \mathrm{~s}$ annealing.

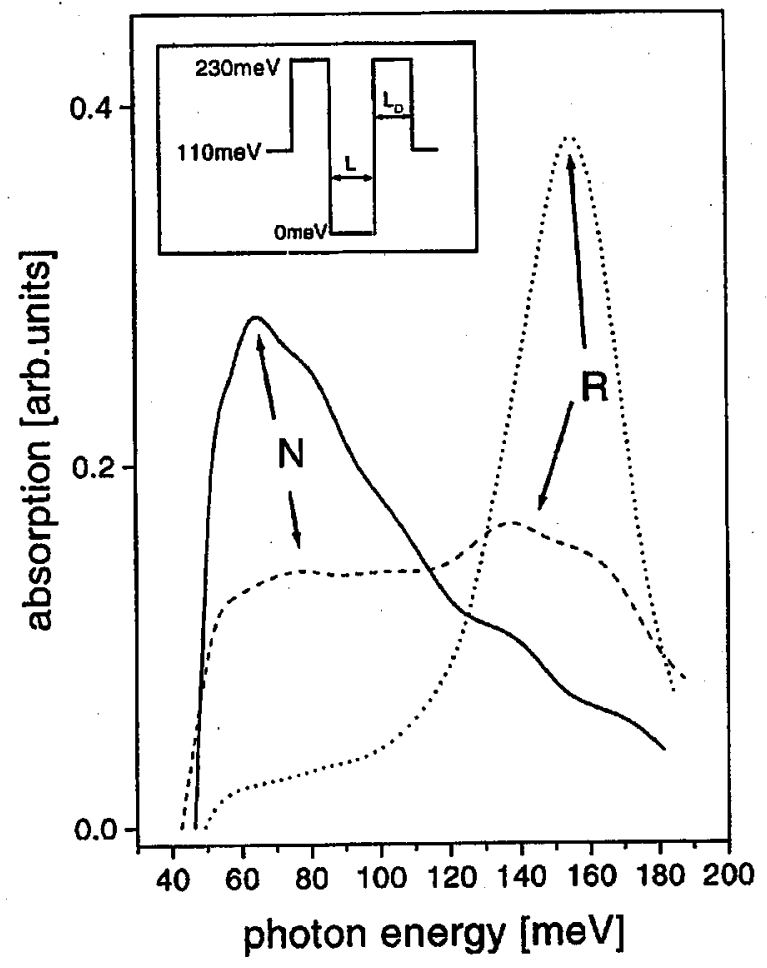

Fig. 2. Bound-to-continuum absorption spectra of three $\mathrm{Al}_{0.15} \mathrm{Ga}_{0.85} \mathrm{As}$ quantum wells ( $L=6 \mathrm{~nm}$ ). with different widths of additional $\mathrm{Al}_{0.3} \mathrm{Ga}_{0.7}$ As barriers $L_{\mathrm{D}}: L_{\mathrm{D}}=1 \mathrm{~nm}$ (solid line), $L_{\mathrm{D}}=2 \mathrm{~nm}$ (dashed line) and $L_{\mathrm{D}}=4 \mathrm{~nm}$ (dotted line). The inset shows schematically the conduction band profile used for the calculation. See the text for the meaning of $(R)$ and $(N)$. 
The curves in Fig. 1 show our results for absorption caused by transitions from the single symmetric bound state to the continuum of antisymmetric envelopes, versus the energy of absorbed photons. We consider here only the $p_{z}$ absorption, where even-even transitions are forbidden for any in-plane wave vector $k_{t}$. As one can see the peak in $\mathrm{B}-\mathrm{C}$ absorption corresponding to transitions to the so-called virtual state $[5,6]$ is moved to lower energies in the cases $(B)$ and $(C)$. In the latter case the second state becomes bound, the absorption maximum is shifted to the lower energies and the oscillator strength of B-C transitions for higher energies is substantially diminished in favour of the $E 1 \rightarrow E 2$ transition and a part of $E 2 \rightarrow C$ ones occurring with photon energies below $20 \mathrm{meV}$ (they are not shown in Fig. 1). These effects, namely red-shifting of absorption maximum and binding of the virtual state are due to the fact that the QW becomes shallower and wider after annealing which may be important for designing infrared detectors.

Next, we study the dependence of the absorption spectrum profile on the width $L_{\mathrm{D}}$ of additional layers of $\mathrm{Al}_{0.3} \mathrm{Ga}_{0.7} \mathrm{As}$ surrounding the well. The band profile of such a structure is shown in the inset of Fig. 2. The spectra for $L_{\mathrm{D}}=$ $1,2,4 \mathrm{~nm}$ are shown in Fig. 2. The low energy maximum $(N)$ occurring for $L_{\mathrm{D}}=$ $1 \mathrm{~nm}$ and $2 \mathrm{~nm}$ originates from the maximum shown in Fig. 1 case $(A)$ (equivalent to $L_{\mathrm{D}}=0$ here). As one can see, when $L_{\mathrm{D}}$ is increased, a resonant peak $(R)$ appears. It corresponds to transitions to the second bound state in $L_{\mathrm{D}} \rightarrow \infty$ limit, which would occur at $145 \mathrm{meV}$. For $L_{\mathrm{D}} \geq 3 \mathrm{~nm}$ the maximum $(R)$ becomes higher than the $(N)^{\prime}$ maximum and its width decreases. The shape of spectrum for $L_{\mathrm{D}} \geq 4 \mathrm{~nm}$ suggests that by appropriate selection of heterostructure components it is feasible to design opto-electronic structures with a selective response based on the $\mathrm{B}-\mathrm{C}$ transitions.

\section{Valence band transitions}

In order to study the intersubband transitions in the VB energy region we consider a $\mathrm{Si} / \mathrm{Si}_{0.8} \mathrm{Ge}_{0.2} / \mathrm{Si}$ rectangular $\mathrm{QW}$ of width $2.2 \mathrm{~nm}$. The VB well consists of three coupled QWs: the heavy hole (HH), light hole (LH) and spin-orbit (SO) hole wells. To consider intersubband transitions we assume that free carriers are present at the VB top, and thus the Fermi level $E_{\mathrm{F}}$ is located below the heavy hole QW bottom (at $75 \mathrm{meV}$, i.e., $k_{\mathrm{F}}=0.1 \mathrm{~nm}^{-1}$ for the first $\mathrm{HH}$ subband). Band profile of the considered structure is given in the inset of Fig. 3. Each of the wells holds only one bound level, the first spin-orbit level (SO1) is immersed in, and coupled with the continua of other wells. For $k_{t}=0$ we are able to distinguish six continua: the two (both parities) HH, two LH-like and two SO-like ones. The SO-like continuum starts $44 \mathrm{meV}$ below the onsets of $\mathrm{HH}$ and $\mathrm{LH}$ continua. For $k_{t} \neq 0$ the bands in the Luttinger-Kohn Hamiltonian are strongly mixed and these distinctions are no longer useful. The problem of defining the energy density of states can only be solved with the method described in the Introduction.

The spectrum consists of B-B type transitions from the only $\mathrm{HH}$ subband (HH1) to the only LH subband (LH1) (below the energy range of our interest), and $\mathrm{HH} 1$ to all continua. For $k_{\mathrm{F}} \simeq 0$ the spectrum profile is similar to a conduction band QW one because $\mathrm{HH}$ subband is almost decoupled from $\mathrm{LH}$ and $\mathrm{SO}$ subbands. 


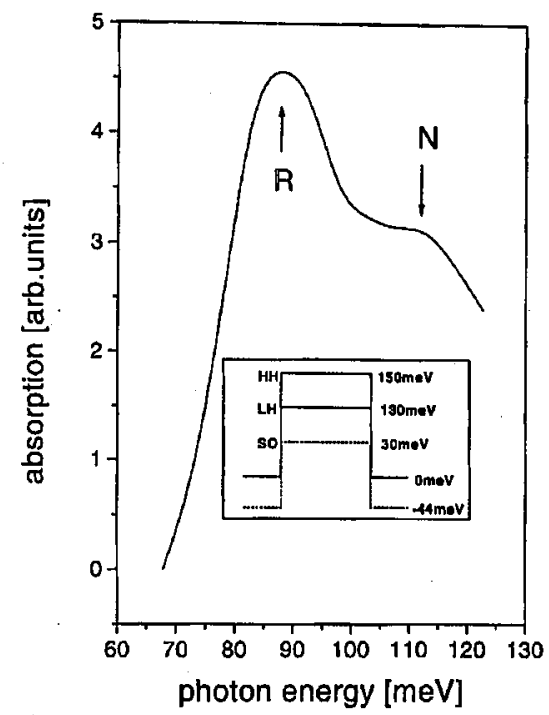

Fig. 3. Bound-to-continuum absorption spectra of $\mathrm{Si} / \mathrm{Si}_{0.8} \mathrm{Ge}_{0.2} / \mathrm{Si}$ quantum well of width $2.2 \mathrm{~nm}$. The inset shows schematically the valence band profile used for the calculation. See the text for the meaning of $(R)$ and $(N)$.

For $k_{\mathrm{F}}=0.1 \mathrm{~nm}^{-1}$ we can distinguish two maxima. The peak at $90 \mathrm{meV}(R)$ is mainly due to $\mathrm{HH} 1 \rightarrow \mathrm{SO} 1$ transitions, its energy lies above the SO continuum onset, but below the $\mathrm{HH}$ and LH one. This is why it can be associated with a resonant SO level coupled to the HH and LH continua. This explanation is confirmed by direct inspection of envelope functions and by the fact that if all the non-diagonal terms of the Luttinger Hamiltonian are set to zero (completely decoupled bands), $\mathrm{HH} 1 \rightarrow \mathrm{SO} 1$ transition energy differs less than $10 \mathrm{meV}$ from the peak $(R)$. The maximum $(N)$ is a remnant of a standard one band B-C absorption profile.

\section{Conclusions}

We have studied intersubband bound-to-continuum transitions in various QW structures. We have shown that when a QW is annealed or narrow lateral barriers are added, the absorption spectra can be substantially modified. A resonance feature in the VB spectrum ( $p$-type $\mathrm{QW}$ ) has been found and interpreted. It is important to note that the VB absorption can be correctly described only within the multiband $k p$ approach.

Additionally we have proven the usefulness of the numerical method we employed for describing the continuum states. We have also proposed a method for calculating the energy density of states, particularly useful when the standard formulae are not applicable.

\section{Acknowledgments}

One of us (R.O.) thanks the Committee for Scientific Research (project No. 2 P03B 124 13) for financial support. 


\section{References}

[1] M. Załużny, Optoelectron. Rev. 3-4, 86 (1995).

[2] G. Fishman, Phys. Rev. B 52, 11132 (1995).

[3] I. Gontijo, T. Krauss, J.H. Marsh, R.M. De La Rue, IEEE J. Quant. Electron. 30, 1189 (1994).

[4] I. Harrison, Semicond. Sci. Technol. 9, 2053 (1994).

[5] V.V. Osipov, F.I. Serzhenko, V.D. Shadrin, Physics and Technics of Semiconductors 23, 809 (1989).

[6] G. Bastard, Wave Mechanics Applied to Semiconductor Structures, Les Editions de Physique, Paris 1988, p. 10. 